\title{
Doppler Radar with In-Band Full Duplex Radios
}

\author{
Seyed Ali Hassani ${ }^{1}$, Karthick Parashar ${ }^{2}$, André Bourdoux ${ }^{2}$, Barend van Liempd ${ }^{2}$ and Sofie Pollin ${ }^{1}$ \\ ${ }^{1}$ Department of Electrical Engineering, KU Leuven, Belgium \\ ${ }^{2}$ Imec, Leuven, Belgium
}

\begin{abstract}
The use of in-band full duplex (IBFD) is a promising improvement over classical TDD or FDD communication schemes. To enable IBFD radios, the electrical balance duplexer (EBD) has been proposed to suppress the direct self-interference (SI) at the RF stage. The remaining SI is typically assumed to be canceled further in the digital domain. In this paper, we show that the non-zero Doppler frequencies can be extracted from the residual SI, giving information about the speed of objects in the environment. As a result, an IBFD radio can be seen as a monostatic Doppler radar which is affected by the communication signal. This paper presents a detailed performance analysis of the different sources of interference affecting the Doppler radar. The performance is also evaluated using a radar-enhanced IBFD prototype consisting of a SDR module and an EBD designed to achieve up to $55 \mathrm{~dB}$ Tx-Rx isolation in the $1.74 \mathrm{GHz}$ RF band. A measurable Doppler component is created by moving a $15 \mathrm{dBsm}$ cone at known velocities at $0.5-1.3 \mathrm{~m}$ from the prototype. For an EBD SI rejection of $45 \mathrm{~dB}$, speeds in the range of 200 to $800 \mathrm{~mm} / \mathrm{s}$ are detected with high accuracy.
\end{abstract}

Index Terms - In-band full duplex, self-interference cancellation, Doppler radar, mono-static wireless sensing.

\section{INTRODUCTION}

Wireless communication is used in almost every electronic device today, and offers a rich functionality when communication is combined with device-level sensors. A smartphone, for example, offers several appealing user experiences as it is typically equipped with a number of integrated sensors, such as a high-resolution camera, barometer, three-axis gyro, accelerometer or GPS. Low-quality sensors can detect movement of the device itself and are quite cheap to integrate. The highquality camera can also detect gestures in the environment, but is a power-hungry and expensive user interface that cannot be used in every device.

Recently, researchers have made progress in developing opportunistic and passive remote radio frequency (RF) sensing techniques which enable remote environmental sensing without adding extra hardware to the device that is often already equipped with a radio. In comparison with the traditional counterparts, e.g., video surveillance and wearable sensors, wireless sensing techniques are often preferred because they preserve the user's comfort and privacy. Employing the ambient electromagnetic signals for gesture detection and localization is a promising direction in this domain.

Given the popularity of Wi-Fi, leveraging Wi-Fi ambient signals to provide radar-like functionality has gained increasing attention in both academia and industry as it can find applications widely ranging from smart home to e-healthcare and through-the-wall human tracking. Several papers have utilized the received signal strength indicator (RSSI) as a metric for indoor localization [1], [2] and passive human activity/gesture recognition [3]-[6]. In fact, Wi-Fi is not the only technology of interest for these applications. For instance, in [7] and [8] the authors propose RSSI-based localization methods which can be applied in a wireless sensor network.

Since the RSSI is easily affected by the temporal and spatial variance due to the multipath effect [9], the newest efforts tend to estimate the Doppler/velocity of the moving target instead. The sensing requirements for the applications mentioned above, however, are not as critical as for those that need a high-frequency wideband Doppler radar. Thus, due to its cost, energy consumption and spectrum occupation, there is no justification to embed such a system into a personal device.

In contrast with an active radar system where a transmitter illuminates a particular signal, a passive Doppler radar relies on an existing electromagnetic signal arriving from two paths: the reference and the surveillance channels. The reference signal consists ideally of the received signal from the line-ofsight path and the surveillance signal involves the reflections from static and moving objects in the vicinity of the transmitter and receiver. Correlating the reference and surveillance signals gives the Doppler frequency shift created by the moving object. The Doppler pattern varies with the movement direction and velocity and hence, one can make use of it to render motion-sensing capability and hand/body gesture recognition.

To perform Doppler measurements with a passive radar, multiple wireless devices are required to extend the angle of view [10]. WiSee [11] makes use of multiple Wi-Fi transmitters and one receiver to enable whole-home sensing and recognition of human gestures via Doppler signature extraction. The presented physical activity sensing solutions in [12]-[15] also employ a Wi-Fi access point (AP) and a dual-channel receiver to perform passive wireless sensing. Chen and his colleagues in [10] improve the accuracy in their activity recognition system using three GPS-based clock synchronized receivers (one reference and two surveillance channels). A simple architecture is also presented in [16] which merely needs one device and a Wi-Fi AP to achieve hand gesture recognition. This system is equipped with a directional coupler and two antennas to collect the surveillance and the reference signals. A mixer then is utilized to extract the Doppler information.

There are two fundamental limitations in these prior art remote sensing techniques:

1) To accomplish radar functionality, the illuminated signal has to be known to the receiver and hence, most of the presented passive schemes need an extra device to 


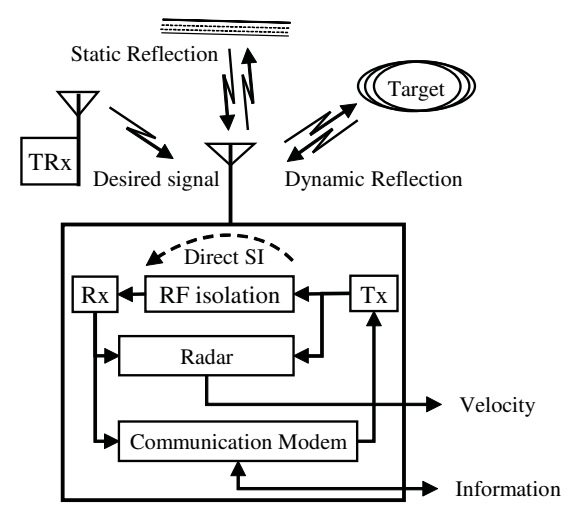

Fig. 1. Overview of the proposed radar-capable IBFD communication device

acquire the reference signal free from reflections and Doppler shifts. This not only increases the realization cost but also poses implementation constraints. For instance, since the ultimate metric appears in the frequency of the estimated Doppler signal, the receivers have to be synchronized to avoid an undesirable local oscillator (LO) frequency shift. Moreover, the use of directional antennas is unavoidable in most of the proposed schemes because the moving target can easily corrupt the reference channel.

2) The transmitted signal in the scenarios mentioned above is not continuous and, hence, another level of synchronization between the illuminator and the receivers is necessary. For example, a synchronization technique is presented in [13] to deal with the noncontinuity of the Wi-Fi signal and reduce the impact of the large beacon interval. Typically these synchronization approaches are accompanied by computation overhead which degrades the performance.

If one can integrate the surveillance receiver and the radar illuminator in one platform, no extra device is needed to collect the reference signal as it can be provided by the transmitter directly. Thus, the impact of the reference channel such as the additive channel noise, undesirable hardware features and lack of channel isolation, would not degrade the radar performance. Furthermore, since both parties are in a single framework, explicit and computationally light synchronization is achievable to mitigate technical difficulties such as long silence intervals. An integrated radar into a single communication device is also more interesting from a cost perspective, as only one set of hardware is required to enable estimation of the Doppler information.

In this paper, we introduce the self-interference cancelation (SIC) technology as a potential solution to combine a Doppler radar and an in-band full duplex (IBFD) communication radio in a single platform. Fig. 1 shows an overview of the proposed system. In the next sections, we explain how the presented system in this figure brings radar-like functionality into an IBFD device merely by employing its already-existing hardware and allows various applications ranging from body/hand gesture detection to onboard Doppler radar.

The remainder of this paper is structured as follows. Section II presents the proposed radar-capable IBFD device by providing some background on IBFD technology. In Section III we develop the system model and discuss various sources of interference followed by a radar performance analysis. The system is then simulated in Section IV and prototyped in Section V. The experimental results are demonstrated in Section VI and, finally, the conclusion is drawn.

\section{RADAR-CAPABLE IBFD WIRELESS DEVICE WITH IDEAL SIC}

This section mainly discusses how to integrate wireless sensing and IBFD technology, to achieve the radar capability by a single device. To this end, we firstly review the principle of IBFD and then explain the proposed radar-capable IBFD architecture relying on an ideal realization of self-interference (SI) rejection.

\section{A. Self-interference Problem in IBFD Communication}

Fig. 1 depicts the proposed transceiver which enables concurrent in-band send and receive. As shown in this figure, there are two replicas of the transmitted signal which loop back into the receiver; a) the direct transmitter SI and b) the reflected interference from the surroundings. The latter is the sum of multiple components resulting from reflections across multiple paths [17].

When the direct transmitter leakage is not attenuated between Tx and Rx ports, it is orders of magnitude stronger than the desired signal coming from a second party communication node, $104 \mathrm{dBm}$ in a typical Wi-Fi scenario for example [18]. Such high power signal saturates the receiver and consequently makes the reception of a desired low power signal impossible. Both the interfering replicas, i.e., the direct and reflected SI, have to be canceled out to preserve the basic functionality of the device. Recent advances in SIC technology enable wireless IBFD systems with high spectrum efficiency. In a typical IBFD architecture, an analog self-interference cancellation (AnSIC) block isolates the Tx and $\mathrm{Rx}$ to reduce the amplitude of the direct SI signal before the analog to digital converter (ADC). This cancellation step plays a vital role as it preserves the ADC dynamic range. Several active AnSIC techniques are reported in the literature, some of which obtain up to $70 d B$ Tx-Rx isolation [19], [20]. To provide adequate signal to noise ratio (SNR) for communication purposes, the remaining SI has to be suppressed to the noise floor. Therefore, in an additional cancellation stage, a digital self-interference cancellation (DiSIC) module estimates the residual SI by modeling the multipath channel and the hardware imperfections [21], [22]. Then, the estimated remaining SI is subtracted from the received signal to achieve $100-110 d B$ total SI rejection.

Surprisingly, the IBFD technology not only doubles the spectrum efficiency but also offers other opportunities such as improving wireless networking by enabling in-band relaying or solving the hidden terminal issue [23]. This paper also aims 


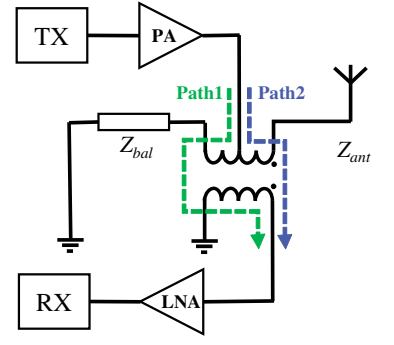

Fig. 2. Structure of a typical EBD-based AnSIC system. High Tx-Rx isolation can be achieved by balancing $Z_{a n t}$ and $Z_{b a l}$ in a way that the two equivalent copies of the transmit signal (through Path 1 and Path 2) reach the receiver port in opposite phase.

to apply this technology to integrate radar-like functionality in a communication device.

\section{B. Electrical Balance Duplexer}

The electrical balance duplexer (EBD) is an interesting SIC solution that addresses the AnSIC problem. Using silicon-oninsulator CMOS technology, the EBD has been realized as an AnSIC module on-chip [19]. Due to its small size, wide center frequency tuning capability, highly-linear characteristics and low insertion loss, the EBD is a potential candidate to be deployed in future communication devices. It is also the key enabling element of the proposed design in this paper as it can sufficiently suppress the direct SI to allow receiving the environmental reflection which is needed for the Doppler measurement.

Fig. 2 illustrates how a hybrid transformer in the EBD chip overcomes the direct leakage through the electrical balancing of two impedances: (a) the antenna impedance $Z_{\text {ant }}$ and (b) the dummy load called the balance network $Z_{b a l}$. The transformer combines the two opposite-phase components to null them out at the receiver port and, at the same time, it allows the environmental signals to reach the receiver. This mechanism relies on tuning the dummy load in a way that it imitates the antenna impedance. Hence, depending on the environmental dynamics affecting the antenna impedance, the $Z_{b a l}$ has to be tuned frequently to maintain the Tx-Rx isolation.

\section{IBFD Radar System Model}

Fig. 3 depicts the system model and the architecture of the proposed IBFD device which also functions as a Doppler radar.

In this model, $X(t)$ and $I_{R}(t)$ are the transmitted signal from the IBFD device and its reflected replica, $I_{S}(t)$ represents the residual SI after analog suppresion and $M(t)$ and $M_{R}(t)$ are respectively the desired signal and its reflections. Besides, the notations $x[n], i_{R}[n], i_{S}[n], m[n]$ and $m_{R}[n]$ are respectively adopted to determine the digital baseband representation of the analog signals described above.

This part explains how the Doppler information can be extracted in an ideal case when the EBD thoroughly rejects the direct SI leakage $I_{S}(t)$ and the DiSIC module precisely estimates the desired signal and its echoed replica.

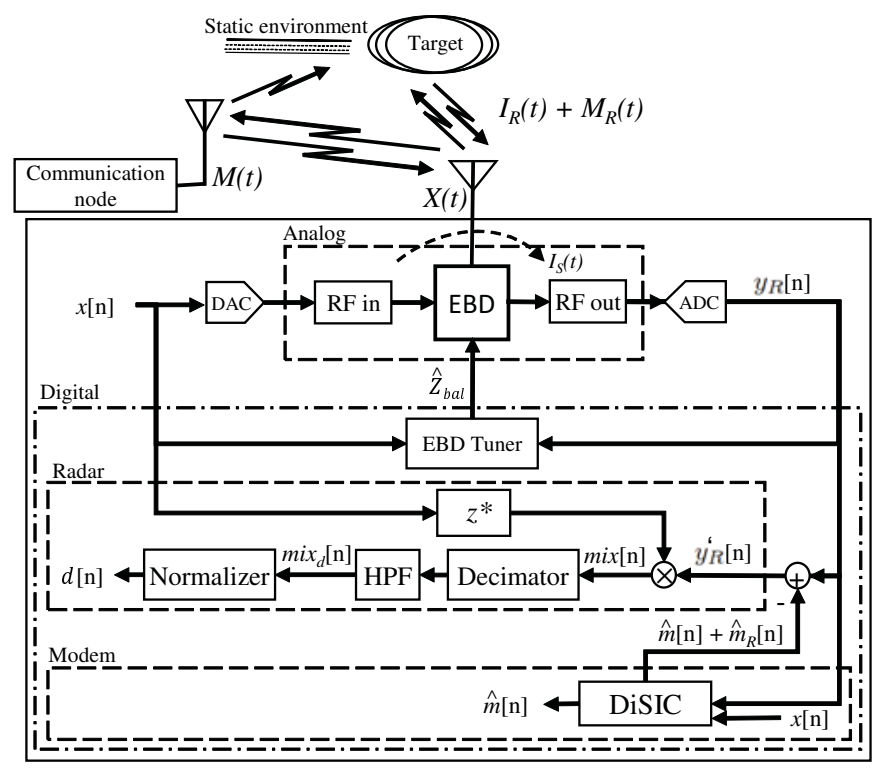

Fig. 3. Proposed architecture of the radar-capable IBFD transceiver.

1) Ideal Mixed Signal: The device transmits the baseband signal $x[n]$ in which $A_{x}[n]$ and $\varphi_{x}[n]$ are respectively the amplitude and phase modulation, i.e.,

$$
x[n]=A_{x}[n] e^{j \varphi_{x}[n]} .
$$

In the context of radar signal processing, the Doppler frequency can be extracted from the product of the transmit signal $x[n]$ with its reflections from the moving target. The baseband reflection $i_{R}[n]$ can be presented as:

$$
i_{R}[n]=A_{E}[n] e^{j\left(\varphi_{x}[n]+w_{d}[n]\right)}+i_{R, s}[n],
$$

where $A_{E}[n]$ is the amplitude of reflected SI signal from the moving target, $w_{d}[n]$ is the Doppler frequency that we are interested to achieve and $i_{R, s}[n]$ is the baseband representation of the superposition of the echoed SI signal from the static environment.

In (2), the phase difference between the transmitted and the reflected signals is assumed negligible over the signal bandwidth as the signal round trip time (RTT) in the applications targeted by this paper ${ }^{1}$ is significantly shorter than the period of the highest transmitted frequency component. For brevity, we also simplify the notations in the rest of the paper by dropping [n] from the sampled baseband signals.

Employing the channel model in [24], the static reflection $i_{R, s}$ in (2) can be expressed as an aggregation of attenuated and phase shifted copies of the transmit signal $x$, i.e.,

$$
i_{R, s}=\sum_{k \in P_{s}} A_{k} e^{j \varphi_{k}} x
$$

${ }^{1}$ Range $<10 m$ and Signal $B W<20 M H z$ 
where $P_{s}$ is the set of dominant reflection paths caused by the static objects in the surroundings, $A_{k}$ represents the constant attenuation for the $k^{\text {th }}$ path with the initial phase offset of $\varphi_{k}$.

The ideal mixed signal $m i x_{i}$ for radar Doppler detection then can be obtained as:

$$
\operatorname{mix}_{i}=x^{*} i_{R}=A e^{j w_{d}}+x^{*} i_{R, s}
$$

where $(*)$ is the complex conjugate operation, (shown by $z^{*}$ in the block diagram) and $A$ is the time-varying amplitude of the ideal mixed signal.

2) Doppler Estimation: The mixed signal has to be decimated to improve the Doppler resolution for a given FFT size. The term $x^{*} i_{R, s}$ in (4) reveals DC behavior that is removed by a high-pass filter (HPF). Assuming a single Doppler component in the reflected SI, the resulting signal $m i x_{d}$ is then normalized to remove the impact of the amplitude modulation. This gives the narrow-band Doppler signal $d$ as follows:

$$
d=\frac{m i x_{d}}{\left|m i x_{d}\right|}=e^{j w_{d}} .
$$

3) Velocity Resolution and Boundaries: We can analyze $d$ to estimate the Doppler frequency $w_{d}$ and the instantaneous radial target velocity (relative to the radar) $v$ :

$$
w_{d}=2 \frac{v}{c} w_{c}
$$

where $c$ is the speed of light and $w_{c}$ is the carrier frequency.

The velocity resolution $v_{\text {res }}$ and the maximum detectable velocity $v_{\max }$ can be determined as:

$$
\begin{gathered}
v_{\text {res }}=\frac{1}{2} \frac{c}{f_{c}} \frac{f_{s}}{N_{d} N_{f f t}}, \\
v_{\max }=\frac{1}{4} \frac{c}{f_{c}} \frac{f_{s}}{N_{d}},
\end{gathered}
$$

where $f_{s}$ is the baseband sampling rate, $N_{d}$ and $N_{f f t}$ are respectively the down-sampling rate and the number of points used to obtain the FFT, and $f_{c}=w_{c} / 2 \pi$ is the carrier frequency.

As the EBD tuning impacts the amplitude and phase of the received signal, the EBD should be stable during the segment used for deriving the Doppler spectrum. The EBD tuning rate $f_{\text {tune }}$ hence limits the minimum detectable velocity $v_{\text {min }}$ in this system. The duration of the segment, determined by the sample rate, decimation factor and FFT size should hence be limited, i.e., $f_{\text {tune }} \leq \frac{f_{s}}{N_{d} N_{f f t}}$. Combining this with (7) we can define the minimum velocity that remains detectable and is given as:

$$
v_{\text {min }} \geq \frac{1}{2} \frac{c}{f_{c}} f_{\text {tune }}
$$

\section{RADAR-CAPABLE IBFD WITHOUT IDEAL SIC}

This section analytically studies the radar performance without ideal SIC, introducing the impact of the multiple interfering components.

\section{A. Mixed Signal with Interference}

The received singal $y_{R}$ in the baseband suffers from two main sources of interference: there is interference from the received message $m$ and its reflections $m_{R}$ and there is the residual direct transmitter leakage $i_{S}$ :

$$
y_{R}=i_{R}+i_{S}+m+m_{R}
$$

\section{B. Removal of the Interfering Components}

To achieve the ideal mixed signal, the radar has to suppress the impact of the interfering components. Below, we discuss how we can remove them using IBFD technology.

1) Removal of Received Interference $m+m_{R}$ : The state of the art DiSIC for IBFD estimates the desired signal and its reflections to enhance the link SNR for communication purposes. These signals can also be subtracted from the received signal $y_{R}$ to improve the quality of the Doppler estimation in the full-duplex mode. An interesting question is to what extent the quality of the DiSIC impacts the radar performance. Thus, we characterize the DiSIC block by its ability to suppress the influence of $m$ and $m_{R}$ in the enhanced received signal $y_{R}^{\prime}{ }^{2}$.

$$
y_{R}^{\prime}=y_{R}-\left(\hat{m}+\hat{m}_{R}\right)=i_{R}+i_{S}+g_{d}\left(m+m_{R}\right),
$$

where $\hat{m}$ and $\hat{m}_{R}$ are the estimated desired signal and its reflection, and $g_{d}=G_{d} e^{j \varphi_{d}}$ is the the complex-valued SI cancelation factor at the digital domain.

2) Suppressing Direct Self-Interference $i_{S}$ : In addition to the interference that is present when the radar is also acting as an IBFD receiver, we have to deal with the self-interference caused by the transmission $x$. This is potentially more challenging as the remaining SI will correlate with the transmitted signal. The residual SI signal $i_{S}$ can be written as:

$$
i_{S}=g_{e} x
$$

where $g_{e}=G_{e} e^{j \varphi_{e}}$ and, $G_{e}$ and $\varphi_{e}$ are respectively the EBD's SI attenuation factor and phase shift.

\section{Doppler Signal Approximation}

To estimate the Doppler signal at this point, we start from the interfered mixed signal that can be determined as:

$$
m i x=x^{*}\left(i_{R}+g_{e} x+g_{d}\left(m+m_{R}\right)\right),
$$

where it is clear that there are two distortion contributions to the mixed signal, one correlating with the transmitted signal and one independent from $x$. In the next section, we will simulate the impact of both sources of interference, but it is already clear that the effect from $m$, which is not correlated and also has low received power, will most likely be small.

\footnotetext{
${ }^{2}$ While the DiSIC block typically only reconstructs $m$, we can assume that this is done using some estimate of the channel for equalization, hence also giving knowledge about $m_{R}$ at the receiver.
} 
Symbols

Simulation 1

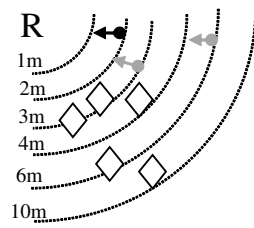

Simulation 2

R Radar

M Transmitter of $m$

$\diamond$ Static reflector

$\leftrightarrow$ Target moving at $2 \mathrm{~m} / \mathrm{s}$

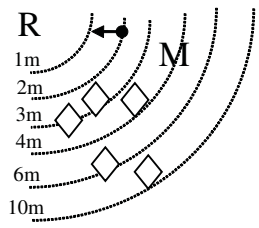

Fig. 4. Two simulated scenarios to evaluate the radar capability of the proposed system.

TABLE I

IEEE 802.11P-LIKE OFDM WAVEFORM CHARACTERISTICS

\begin{tabular}{|c|c||c|c|}
\hline Param. & Value & Param. & Value \\
\hline strength & $20 \mathrm{dBm}$ & OFDM subcarriers & 54 \\
\hline bandwidth & $10 \mathrm{MHz}$ & OFDM pilots & 4 \\
\hline bit rate & $3 \mathrm{Mbps}$ & modulation & QPSK \\
\hline
\end{tabular}

Besides, it is obvious from (13) that the radar has to minimize $G_{e}$ and $G_{d}$ to approach the ideal mixed signal defined in (4).

Similar to the reflection from static objects $i_{R, s}$, the decimated remaining direct self-interference $i_{S}$ will create a Doppler component at DC frequency that is removed by the DC removal filter.

Regarding the presented model, it is worthwhile to note that in a half-duplex scenario, there is no desired signal $m$ in the transmission time which means the signal $y_{R}$ exclusively comprises one source of interference; the residual direct SI. Hence, an EBD would be the only required deployment to provide radar capability for a communication device, and there is no need for a DiSIC step.

\section{Simulation RESUlts}

In this section, we simulate the presented system in the presence of both interfering sources to investigate the model performance as a Doppler radar. This part also aims to verify that the interference from the received signal $m$ can be neglected and no intensive DiSIC processing is needed for radar performance. In this simulation, we chose an OFDM waveform for both the transmitted signal $x$ and received message $m$, using parameters as specified in Table I for IEEE 802.11 p to modulate two statistically independent and zeromean random sets of data. The simulation is carried out in Matlab in double precision floating-point, the in-band thermal noise is assumed to be $-90 \mathrm{dBm}$ and the IBFD receiver samples at $30 \mathrm{MHz}$. The received signal at the EBD output is computed taking into account all interfering components as determined in (13). The mixed signal is then decimated to obtain $0.5 \mathrm{sec}$ of IQ samples at $457 \mathrm{~Hz}$. In the next step, the Doppler signal is estimated and windowed (Hanning) to generate the FFT components. Theoretically, the obtained FFT yields $12.4 \mathrm{~mm} / \mathrm{s}$ velocity resolution and the maximum detectable velocity of $\pm 14.23 \mathrm{~m} / \mathrm{s}$ at $2.4 \mathrm{GHz}$ RF band.

Fig. 4 depicts the two simulated scenarios where five static reflectors are positioned in $3-10 \mathrm{~m}$ around the radar enabled

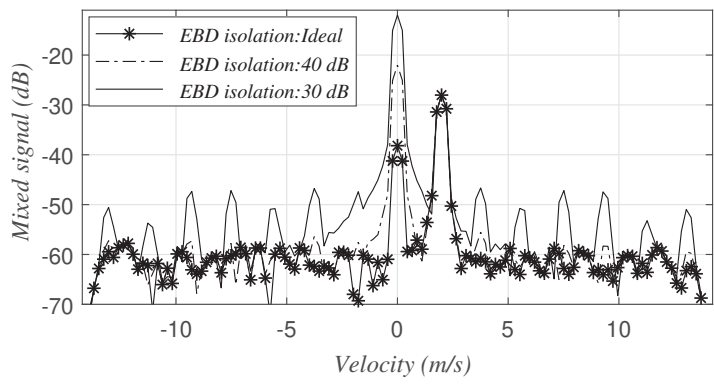

Fig. 5. Spectrum of the mixed signal for different levels of EBD Tx-Rx isolation in the absence of the desired signal $m$.

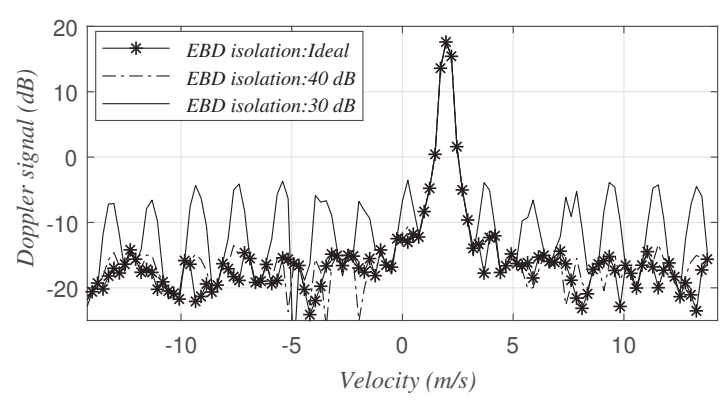

Fig. 6. Spectrum of the estimated Doppler signal for different levels of EBD Tx-Rx isolation in the absence of the desired signal $m$.

IBFD device to resemble an indoor multipath channel. A target is also involved which moves at $2 \mathrm{~m} / \mathrm{s}$ towards the radar.

\section{A. Radar Performance without the Desired Signal $m$}

In the first simulation, the influence of the EBD SI rejection is evaluated in the absence of the intended message $m$. Fig. 5 shows the spectrum of the resulting mixed signal when the target is at $2 \mathrm{~m}$. This figure reveals that reducing the EBD isolation $\left(-G_{e}(d B)\right)$ not only increases the DC component as the direct SI has a zero Hz Doppler, but also creates ambiguity peaks in the mixed signal. Importantly, the Doppler component maintains the same level. This simulation shows that an EBD isolation better than $40 \mathrm{~dB}$ can, for the simulated scenario, sufficiently remove the noise on the mixed signal. Fig. 6 also illustrates how the DC removal filter eliminates the strong DC component from the estimated Doppler signal $d$.

By comparing both ideal and non-ideal Doppler signals, we can determine a normalized SNR as:

$$
S N R_{\text {norm }}=S N R_{\text {est }}-S N R_{\text {opt }},
$$

where $S N R_{\text {opt }}$ and $S N R_{\text {est }}$ are the SNR of the estimated Doppler resulting respectively from the ideal mixed signal in (4) and the interfered mixed signal in (13).

This test is continued by positioning the target at different distances $(2 \mathrm{~m}, 3 \mathrm{~m}$ and $6 \mathrm{~m})$ to also assess the detection range of the system. Fig. 7 shows the resulting $S N R_{\text {norm }}$ for various levels of EBD performance. It is evident that increasing the EBD SI cancellation improves the quality of 


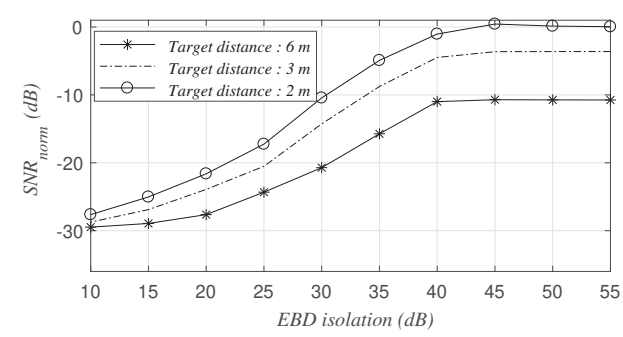

Fig. 7. The normalized SNR of the estimated Doppler signal for different levels of EBD isolation and various distances of the moving target in the absence of the desired signal $m$.

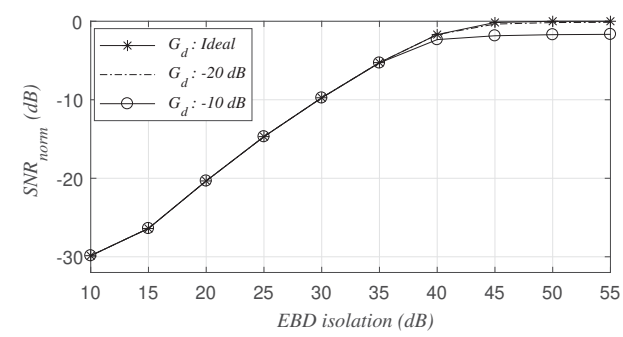

Fig. 8. Normalized SNR of the estimated Doppler signal for different levels of EBD isolation and various performance of the DiSIC block.

the Doppler signal in all the cases. Our simulation also shows that an analog suppression of the self-interference of $40 \mathrm{~dB}$ is sufficient independent of the target distance. Moreover, the results reveal that the system produces better $S N R_{n o r m}$ as the target approaches the IBFD radio.

\section{B. Radar Performance with the Desired Signal $m$}

In the second simulation scenario, we locate the transmitter of the desired signal at $4 \mathrm{~m}$ from the radar and generate the corresponding reflections (see Fig. 4). Fig. 8 represents a similar curve to assess the significance of the DiSIC parameter $G_{d}$ suppressing the interference from the received message $m$. As expected, $m$ and its environmental reflections do not impact the radar performance of the system as $m$ and $x$ are uncorrelated. Nevertheless, the DiSIC block can enhance the radar performance when $m$ is significantly stronger than the reflection $i_{R}$, which is visible in Fig. 8, when the EBD isolation is above $45 d B$.

\section{RADAR-CAPABLE IBFD PROTOTYPE}

In this section, we introduce our IBFD prototype used on an EBD for the analog self-interference cancellation. Fig. 9 (Left) illustrates the function schematic of the prototyped device. As our simulation results confirmed that the main source of interference for the Doppler signal is the leaked direct SI, subject to the performance of the EBD, we focus on this source of interference in our experiments. This also simplifies the prototype as there is no need for a DiSIC block. To the best of our knowledge, it is the first demonstration of EBDbased mono-static Doppler radar which is integrated into a communication device.

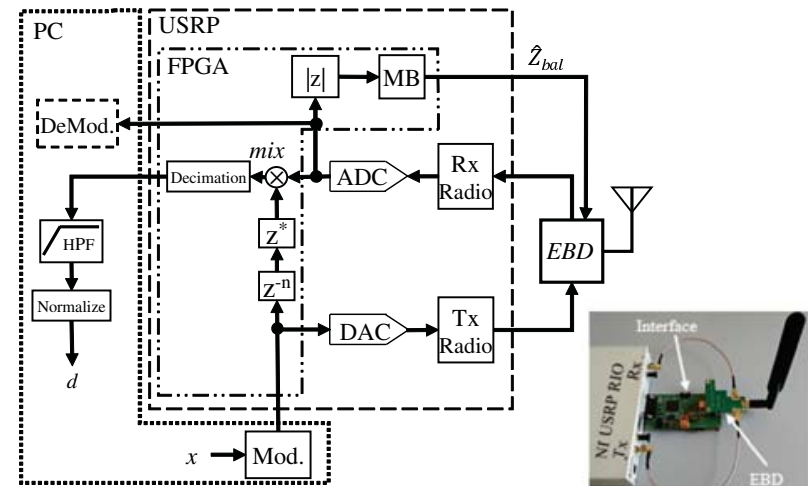

Fig. 9. Block diagram (Left) and the picture (Right) of the prototyped radarcapable communication device.

Fig. 9 (Right) shows the picture of the prototype which consists of one SDR, one customized EBD-based AnSIC module [20] and one PC. The SDR is a NI USRP RIO with a Kintex-7 FPGA onboard. The EBD is connected to the USRP by two $25 \mathrm{~cm}$ RF cables and a GPIO bus. The RF cables connect the EBD and the USRP's receiver and transmitter. The radio front-end ranges from $400 \mathrm{MHz}$ to $4.4 \mathrm{GHz}$ and samples at $120 \mathrm{MSamples} / \mathrm{s}$. The $Z_{b a l}$ in the prototyped EBD comprises four adjustable capacitors, each can obtain $2^{8}$ distinct capacitance values. To tune the EBD and achieve adequate SI rejection, we use a particle swarm (PS) algorithm [25] which is implemented on a MicroBlaze softcore [26] deployed on the FPGA (shown by MB block in Fig. 9). Running at $150 \mathrm{MHz}$, the MicroBlaze allows EBD tunning within $1 \mathrm{~ms}$ through the GPIO bus. The whole setup can be controlled and monitored via a PCI-e connection and a LabVIEW user interface. Regarding the EBD's maximum tolerable RF input and its operating frequency band, we decrease the power of the OFDM signal (see table I) to $5 \mathrm{dBm}$ and tune the USRP's Tx and $\mathrm{Rx}$ at $1.74 \mathrm{GHz}$. We also use an omnidirectional antenna as such the device can radiate/receive to/from all directions and maintain its communication functionality. To compensate the Tx-baseband to Rx-baseband delay, the model employs an n-tap delay block shown by $z^{-n}$ in the block diagram and the measured $n$ is approximately 119 samples in our prototype. The USRP produces $120 \mathrm{MHz}$ complex baseband signal which has to be mixed with the transmitted signal. The resulting signal is then decimated to achieve narrow-band (457 Hz) complex mixed signal. The real-time processing on the FPGA is in 16-bit and 32-bit fixed-point precision and, as shown in Fig. 9, the rest of the computation is accomplished on a computer to obtain the micro-Doppler profile which theoretically enables detecting up to $\pm 19.64 \mathrm{~m} / \mathrm{s}$ motions with $10.6 \mathrm{~mm} / \mathrm{s}$ resolution.

\section{EXPERIMENTAL RESULTS}

This section details the experimental results obtained with the IBFD prototype with analog SIC. First, the required EBD tuning rate is measured in an indoor environment. Second, we evaluate the impact of fixing the EBD state when computing 


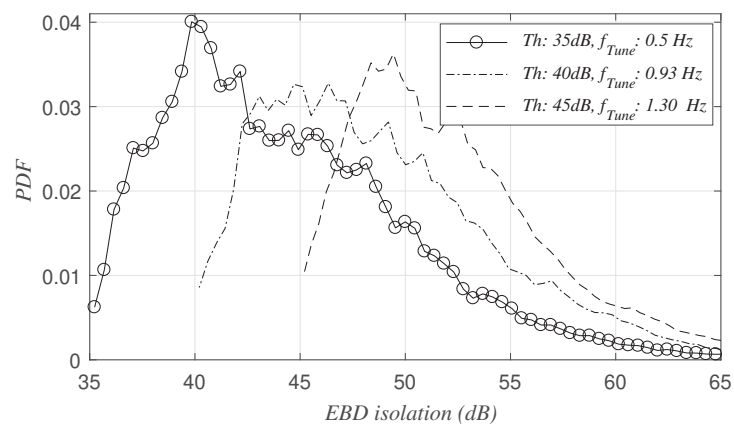

Fig. 10. Probability distribution of the EBD isolation performance for various tuning thresholds, measured in an indoor environment.

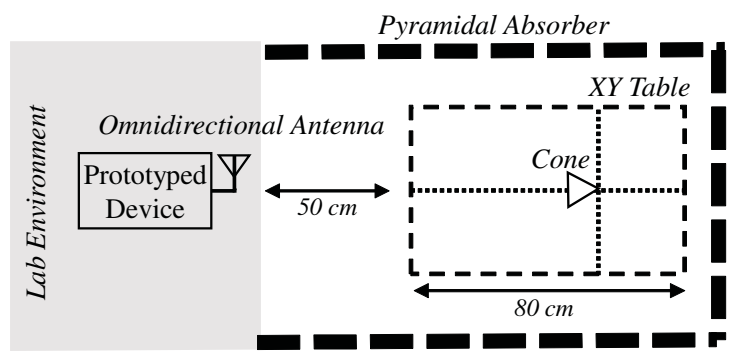

Fig. 11. Schematic of the testbed.

the Doppler spectrum. We also assess the accuracy of the velocity estimation experimentally.

\section{A. EBD Tuning Trade-off}

In Section II, we discussed the necessity of EBD retuning and introduced $f_{\text {tune }}$ as a parameter which limits the minimum detectable velocity. In this part, we investigate $f_{\text {tune }}$ in an indoor environment. Fig. 10 displays the probability distribution of the EBD isolation, measured in a typical office room where the device is placed on a desk next to a working person. In each experiment, an isolation threshold is determined to trigger the EBD tunning algorithm. The level of the SI and the number of triggers are recorded for 60 minutes to estimate how often the EBD has to be tuned to maintain the Tx-Rx isolation below the threshold. It is evident that increasing the isolation threshold raises the required EBD tuning speed and, consequently, increases the minimum detectable velocity determined in (9). According to the experimental results in Fig. 10, to obtain $45 d B, 40 d B$ and $35 d B$ analog SI cancellation, the EBD requires respectively $1.3 \mathrm{~Hz}, 0.93 \mathrm{~Hz}$ and $0.5 \mathrm{~Hz}$ tuning rates. These tunning frequencies limit $v_{\min }$ to 111,80 and $43 \mathrm{~mm} / \mathrm{s}$ respectively. We note that the actual achieved isolation depends on the EBD setting and the dynamic environment, giving a distribution of instantaneous isolation values. Each time the EBD is retuned, a more optimal configuration for the given environment is determined.

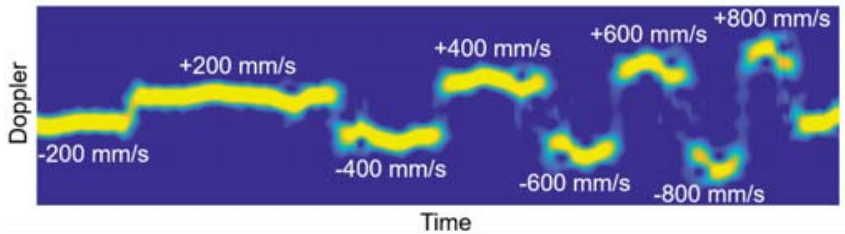

Fig. 12. Measured Doppler profile produced by the prototype device presenting the movement of a mounted cone reflector on a XY table. STFT segment $800 \mathrm{~ms}$ with $85 \%$ overlap, Hanning window, 8k-point FFT, EBD isolation $>45 d B$.

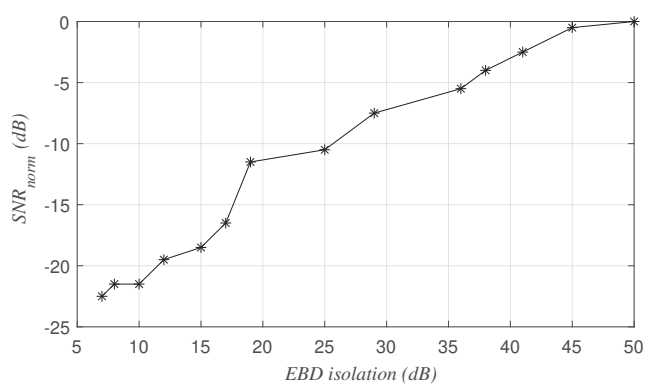

Fig. 13. Measured influence of the EBD isolation on radar capability of the proposed system.

\section{B. Doppler Measurement}

To evaluate the radar capability of the prototyped device, a remotely controlled $\mathrm{XY}$ table is utilized to move a cone reflector with radar cross section (RCS) $15 \mathrm{dBsm}$ at different speeds ranging from 200 to $800 \mathrm{~mm} / \mathrm{s}$, as shown in Fig. 11 . Due to the omnidirectionality of the antenna, the system is also exposed to statistic reflections from a lab environment.

Fig. 12 shows the Doppler profile obtained by short-time Fourier transform (STFT), when the reflector moves forward and backward at different velocities. Each segment in this spectrogram is $800 \mathrm{~ms}$ including $85 \%$ overlap with the adjacent frame. There is a visible artifact in the spectrogram when the XY table changes the direction of the target. It is due to inertia in the mechanical setup.

In the next experiment, $S N R_{\text {norm }}$ of the Doppler signal is measured for different levels of analog SI cancellation while the target moves at $200 \mathrm{~mm} / \mathrm{s}$. The result in Fig. 13 reveals that improving the EBD SI isolation enhances the estimated Doppler signal. Besides, for higher levels of EBD SI rejection, this curve also behaves similar to the simulation results in Fig. 7 and Fig. 8.

Fig. 14 presents the performance in terms of the probability of correct velocity detection $\left(P_{d}\right)$ and the likelihood of false alarm $\left(P_{f a}\right)$. For each measurement in this figure, the reflector moves (forward and backward) five times at the speed of $200 \mathrm{~mm} / \mathrm{s}$. The measurements are performed for various levels of EBD SI cancellation. Then, the spectrogram is formed by a 365-point FFT and the largest FFT component determines the target velocity. The estimated value is then compared with the actual target speed. By changing the detection thresholds, various probabilities are measured. Regarding the results in 


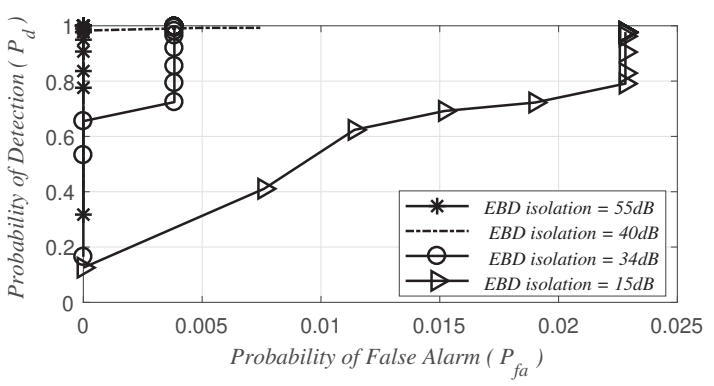

Fig. 14. Measured $P_{d}-P_{f a}$ graph for different levels of EBD SI rejection.

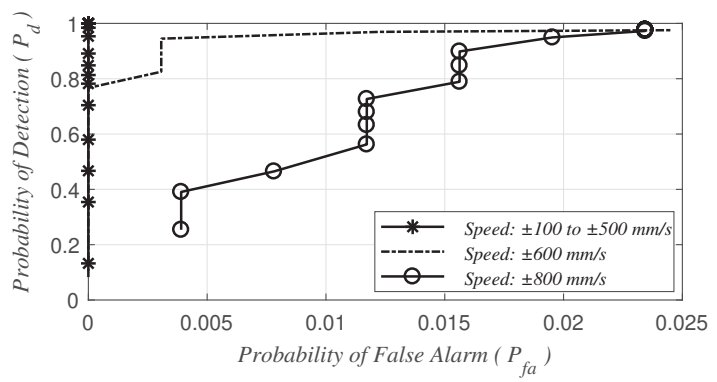

Fig. 15. Measured $P_{d}-P_{f a}$ graph for different velocities, EBD isolation $>$ $45 d B$.

Fig. 14, increasing the EBD SI suppression improves the probability of correct velocity measurement.

The same strategy is adopted to assess the velocity measurement capability of the system when the EBD isolation is maintained $>45 d B$. Fig. 15 illustrates the $P_{d}-P_{f a}$ graph for different speeds. In this test, we consider the measurement tolerance of $\pm 4 v_{\text {res }}$ to deal with the artifact caused by the vibration at the moment the cone changes the movement direction. This experimental result also shows that the device allows high precision velocity detection during signal transmission, as all experiments are carried out with modulated waveforms.

\section{CONCLUSION}

This paper demonstrates the ability to integrate the functionality of a mono-static Doppler radar in an IBFD-capable communication device. We study how various interfering sources influence the performance of the integrated Doppler radar. When the radar is sensing movements in the environment while transmitting and receiving messages in in-band full duplex mode, our simulation results show that the impact of an interfering communication device is found to be negligible. This is owing to the fact that the communication signal is largely uncorrelated and a typical DiSIC module can overcome this source of interference.

The proposed concept is also prototyped using a commercial off-the-shelf SDR platform and a custom made EBD at $1.74 \mathrm{GHz} \mathrm{RF}$ band. Using a moving reflector mounted on a $\mathrm{XY}$ table, the prototyped radar-capable communication device is evaluated by investigating the influence of the EBD Tx-Rx isolation and the velocity measurement in the term of false alarm and detection probabilities. The experimental results prove that an analog self-interference cancellation of $45 d B$ is able to sufficiently cancel the self-interference on the Doppler signal and achieves high-resolution velocity estimation in an indoor environment. The proof-of-concept prototype could precisely measure velocities in the range of $200-800 \mathrm{~mm} / \mathrm{s}$.

The radar range resolution, however, is very poor as the communication standards primarily dictate the operation bandwidth. This implies that it can only effectively be used as a Doppler radar.

Using radars for human activity detection has been popularly adopted in recent years. The realization of the Doppler radar functionality in the communication device presents several opportunities such as near range gesture recognition without having to necessarily operate another RF device. To the best of our knowledge, this is the first of its kind to describe and also prototype a device that can potentially be used for both communication and extraction of Doppler features simultaneously. Going further, future work entails a more detailed analysis of the trade-off between EBD tuning accuracy and speed, versus the range of Doppler frequencies that can be detected in a variety of scenarios.

\section{ACKNOWLEDGMENT}

This work was funded by the European Union's Horizon 2020 under grant agreement no. 732174 (ORCA project). We would also like to thank Professor F. Horlin, University of Brussels (ULB), for his helpful comments.

\section{REFERENCES}

[1] X. Zhu and Y. Feng, "RSSI-based algorithm for indoor localization," Communications and Network, vol. 5, no. 02, p. 37, 2013.

[2] X. Luo, W. J. OBrien, and C. L. Julien, "Comparative evaluation of received signal-strength index (RSSI) based indoor localization techniques for construction jobsites," Advanced Engineering Informatics, vol. 25, no. 2, pp. 355-363, 2011

[3] H. Abdelnasser, M. Youssef, and K. A. Harras, "Wigest: A ubiquitous wifi-based gesture recognition system," in Computer Communications (INFOCOM), 2015 IEEE Conference on. IEEE, 2015, pp. 1472-1480.

[4] S. Sigg, U. Blanke, and G. Troster, "The telepathic phone: Frictionless activity recognition from WIFI-RSSI," in Pervasive Computing and Communications (PerCom), 2014 IEEE International Conference on. IEEE, 2014, pp. 148-155.

[5] L. Sun, S. Sen, D. Koutsonikolas, and K.-H. Kim, "Widraw: Enabling hands-free drawing in the air on commodity WIFI devices," in Proceedings of the 21st Annual International Conference on Mobile Computing and Networking. ACM, 2015, pp. 77-89.

[6] B. Kellogg, V. Talla, and S. Gollakota, "Bringing gesture recognition to all devices." in NSDI, vol. 14, 2014, pp. 303-316.

[7] G. Mao, B. Fidan, and B. D. Anderson, "Wireless sensor network localization techniques," Computer networks, vol. 51, no. 10, pp. 2529 2553, 2007.

[8] P. Biswas and Y. Ye, "Semidefinite programming for ad hoc wireless sensor network localization," in Proceedings of the 3rd international symposium on Information processing in sensor networks. ACM, 2004, pp. 46-54.

[9] K. Wu, J. Xiao, Y. Yi, M. Gao, and L. M. Ni, "Fila: Fine-grained indoor localization," in INFOCOM, 2012 Proceedings IEEE. IEEE, 2012, pp. $2210-2218$

[10] Q. Chen, B. Tan, K. Chetty, and K. Woodbridge, "Activity recognition based on micro-doppler signature with in-home Wi-Fi," in e-Health Networking, Applications and Services (Healthcom), 2016 IEEE 18th International Conference on. IEEE, 2016, pp. 1-6. 
[11] Q. Pu, S. Gupta, S. Gollakota, and S. Patel, "Whole-home gesture recognition using wireless signals," in Proceedings of the 19th annual international conference on Mobile computing \& networking. ACM, 2013, pp. 27-38.

[12] B. Tan, K. Woodbridge, and K. Chetty, "A real-time high resolution passive WiFi doppler-radar and its applications," in Radar Conference (Radar), 2014 International. IEEE, 2014, pp. 1-6.

[13] W. Li, B. Tan, R. J. Piechocki, and I. Craddock, "Opportunistic physical activity monitoring via passive WiFi radar," in e-Health Networking, Applications and Services (Healthcom), 2016 IEEE 18th International Conference on. IEEE, 2016, pp. 1-6.

[14] W. Li, B. Tan, and R. J. Piechocki, "Non-contact breathing detection using passive radar," in Communications (ICC), 2016 IEEE International Conference on. IEEE, 2016, pp. 1-6.

[15] B. Tan and K. Woodbridge, "A wireless passive radar system for real-time through-wall movement detection," IEEE Transactions on Aerospace and Electronic Systems, vol. 52, no. 5, pp. 2596-2603, 2017.

[16] M.-C. Tang, F.-K. Wang, and T.-S. Horng, "Human gesture sensor using ambient wireless signals based on passive radar technology," in Microwave Symposium (IMS), 2015 IEEE MTT-S International. IEEE, 2015, pp. 1-4

[17] D. Kim, H. Lee, and D. Hong, "A survey of in-band full-duplex transmission: From the perspective of PHY and MAC layers," IEEE Communications Surveys \& Tutorials, vol. 17, no. 4, pp. 2017-2046, 2015.

[18] A. Sabharwal, P. Schniter, D. Guo, D. W. Bliss, S. Rangarajan, and R. Wichman, "In-band full-duplex wireless: Challenges and opportunities." IEEE Journal on Selected Areas in Communications, vol. 32, no. 9 , pp. 1637-1652, 2014

[19] B. van Liempd, B. Hershberg, S. Ariumi, K. Raczkowski, K.-F. Bink, U. Karthaus, E. Martens, P. Wambacq, and J. Craninckx, "A+ 70dbm iip3 electrical-balance duplexer for highly integrated tunable frontends," IEEE Transactions on Microwave Theory and Techniques, vol. 64, no. 12 , pp. 4274-4286, 2016.

[20] T. Vermeulen, B. van Liempd, B. Hershberg, and S. Pollin, "Real-time RF self-interference cancellation for in-band full duplex," in Dynamic Spectrum Access Networks (DySPAN), 2015 IEEE International Symposium on. IEEE, 2015, pp. 275-276.

[21] D. Korpi, Y.-S. Choi, T. Huusari, L. Anttila, S. Talwar, and M. Valkama, "Adaptive nonlinear digital self-interference cancellation for mobile inband full-duplex radio: Algorithms and RF measurements," in Global Communications Conference (GLOBECOM), 2015 IEEE. IEEE, 2015, pp. 1-7.

[22] D. Korpi, J. Tamminen, M. Turunen, T. Huusari, Y.-S. Choi, L. Anttila, S. Talwar, and M. Valkama, "Full-duplex mobile device: Pushing the limits," IEEE Communications Magazine, vol. 54, no. 9, pp. 80-87, 2016.

[23] T. Vermeulen, F. Rosas, M. Verhelst, and S. Pollin, "Performance analysis of in-band full duplex collision and interference detection in dense networks," in Consumer Communications \& Networking Conference (CCNC), 2016 13th IEEE Annual. IEEE, 2016, pp. 595-601.

[24] D. Tse and P. Viswanath, Fundamentals of wireless communication. Cambridge university press, 2005.

[25] R. Eberhart and J. Kennedy, "A new optimizer using particle swarm theory," in Micro Machine and Human Science, 1995. MHS'95., Proceedings of the Sixth International Symposium on. IEEE, 1995, pp. $39-43$

[26] Xilinx

"Microblaze soft

processor," http://www.xilinx.com/tools/microblaze.htm 